\title{
Shortcomings/limitations of Blockwise Granger Causality and Advances of Blockwise New Causality
}

\author{
Sanqing Hu, Senior Member, IEEE, Xinxin Jia, Jianhai \\ Zhang, Wanzeng Kong, Yu Cao, Senior Member, IEEE
}

\begin{abstract}
Multivariate blockwise Granger causality (BGC) is used to reflect causal interactions among blocks of multivariate time series. Especially, spectral BGC and conditional spectral BGC are used to disclose blockwise causal flow among different brain areas in various frequencies. In this paper, we demonstrate that (i) BGC in time domain may not necessarily disclose true causality. (ii) Due to the use of the transfer function or its inverse matrix and partial information of the multivariate linear regression model, both of spectral BGC and conditional spectral BGC have shortcomings and/or limitations which may inevitably lead to misinterpretation. We then in time and frequency domains develop two new multivariate blockwise causality methods for the linear regression model, called blockwise new causality (BNC) and spectral BNC respectively. By several examples we confirm that $\mathrm{BNC}$ measures are more reasonable and sensitive to reflect true causality or trend of true causality than BGC or conditional BGC. Finally, for EEG data from an epilepsy patient we analyze event-related potential (ERP) causality and demonstrate that both of BGC and BNC methods show significant causality flow in frequency domain, but the spectral BNC method yields satisfactory and convincing results which are consistent with eventrelated time-frequency power spectrum activity. The spectral BGC method is shown to generate misleading results. Thus, we deeply believe that our new blockwise causality definitions as well as our previous new causality definitions in [30] may have wide applications to reflect true causality among two blocks of time series or two univariate time series in economics, neuroscience and engineering.
\end{abstract}

Index Terms-Blockwise Granger causality (BGC), Blockwise new causality (BNC), ERP, Multivariate linear regression model, Power spectrum.

\section{INTRODUCTION}

The issue of causality has been an important topic for over two thousand years. Along this line researchers defined many causality methods to reveal causal influence in one system. To judge whether one causality method is good or bad, the key point is that the causality method can well reveal the inherent

This work was supported in part by the National Natural Science Foundation of China under Grant 61473110 and Grant 61100102, in part by the Natural Science Foundation of Zhejiang Province, China, under Grant LZ13F030002, in part by the International Science and Technology Cooperation Program of China under Grant 2014DFG12570, and in part by the U.S. National Science Foundation under Grant 1156639 and Grant 1229213. (Corresponding author:Jianhai Zhang.)

S. Hu, X. Jia, J. Zhang, and W. Kong are with College of Computer Science, Hangzhou Dianzi University, Hangzhou, 310018 China (email:sqhu@hdu.edu.cn; jiaxin07127@163.com; jhzhang@hdu.edu.cn; kongwanzeng@gmail.com).

Y. Cao is with Department of Computer Science, The University of Massachusetts Lowell, MA 01854 USA (email: ycao@cs.uml.edu). true (real) causality in the system. In the literature, GC is one of the most popular methods to detect the directional causal influence of system components because of its simplicity and easy implementation, and plays a vital role in knowing about systems behavior in a variety of areas, such as economics [1], [2], climate studies [3], genetics [4], and neuroscience [5], [6]. In 1956, Wiener [7] proposed the basic idea of GC and in 1969, Granger [8] formalized GC with a linear regression model of time series, which can be described below: if the variance of the prediction error of one variable $X_{1}$ is larger than or equal to that of the prediction error by adding the second variable $X_{2}$, then $X_{2}$ has a causal flow on $X_{1}$. Although GC has tremendous applications in many areas, it has also been criticized by many researchers for a variety of reasons [9], [10]. The criticism has mostly been focused on the debate on the relationship between GC and true causality. Moreover, Granger himself indicates that GC may not necessarily be true causality [11]. The original GC in time domain is only suitable for the causality between pairs of variables in a multivariate set. To analyze causality for multivariate time series, conditional GC has been developed [12], [13]. To discuss causality in some specific frequency, the original GC has been developed in spectral domain such as spectral GC and conditional spectral GC [12], [13]. Some other typical spectral causality measures include partial directed coherence (PDC) [14], relative power contribution (RPC) [15], directed transfer function (DTF) [16] and short-time direct directed transfer function (SdDTF) [17]. These measures have yielded many interesting results in the applications to neural data [12], [15], [17]-[29]. The reason why these related measures were developed is precisely because there is no single accepted definition for true causality, and these alternative measures reflect alternative notions of causality.

In 2011, Hu et. al [30] listed many shortcomings/limitations of GC or Granger-like causality methods by using a number of illustrative examples and showed that GC is only a causality definition in the sense of Granger and why GC does not reflect real causality, and meanwhile proposed new causality (NC). $\mathrm{Hu}$ et. al [30] defined $\mathrm{NC}$ as a causality from one time series $(Y)$ to another time series $(X)$, represented in the multivariate time series linear regression model. The key idea of NC is to use the proportion that $Y$ occupies among all contributions to $X$ to describe the strength of the real causality from $Y$ to $X$, and is shown to be more accurate and sensitive than GC to reveal true causality or trend of true causality by those 
examples. By one simple example $\mathrm{NC}$ is shown to be an extension of GC and overcomes GC's shortcomings /limitations. For a multivariate time series linear regression model, $\mathrm{Hu}$ et. al [30] gave a general guideline to define causality from $Y$ to $X$, that is, one must consider all contributions to $X$. A good definition for defining real causality should describe that, among all contributions to $X$, that percentage (or the proportion) that $Y$ contributes (or occupies). It is obvious that the true causality is larger when the proportion is larger or vice versa. Any causality measure violating this general guideline inevitably cannot reflect true causality. For example, the majority of existing causality definitions in the spectral domain are based on the linear regression model's transfer function matrix or the inverse matrix of the transfer function. The transfer function matrix is totally different from the linear regression model's coefficient matrix by noting the difference of the corresponding diagonal elements of two matrices. Therefore, they all violate the general guideline and inevitably cannot reflect true causality. In our recent work [31] we applied NC and GC methods to study causality flow during motor imagery using scalp EEGs in BCI competition IV held in 2008 and found that NC method largely improves the classification rate than GC method. It is noted that $\mathrm{NC}$ can be used for multivariate time series unlike the bivariate GC which can only be applied to two time series although conditional GC can be applied to three or more time series.

It should be emphasized that any causality measure based on multivariate linear regression model with time-invariant coefficients is causal effect or causal influence and is not causal mechanism. In [32] the authors pointed out that $\mathrm{Hu}$ et al's NC [30] is not causal effect. Instead, it is causal mechanism because the $\mathrm{NC}$ defined by $\mathrm{Hu}$ et al [30] employs the linear regression model's time-invariant coefficients. GC is causal effect because there is the "effect": reduction of prediction error. We argue that this statement in [32] is not correct. The reasons are as follows: i) Causal effect and causal mechanism are two different concepts. The influence from one variable $X_{2}$ to the other variable $X_{1}$ is called causal effect or causal influence. Causal mechanism represents a process through which the treatment/input causally affects the outcome/observation (see, e.g., the diagram of a simple causal mechanism in Figure 1 of [33]. According to this diagram, dynamical causal model (DCM) is a typical causal mechanism). Let's see DCM's equivalent discrete form EGCM of equation (2) in [34] in the case of $p=1$ and $g(x)=x$, that is, the following GCM with additional inputs:

$$
\left\{\begin{aligned}
X_{1, t} & =a_{11} X_{1, t-1}+\left[a_{12}+u_{t-1}\right] X_{2, t-1}+\eta_{1, t} \\
X_{2, t} & =a_{21} X_{1, t-1}+\left[a_{22}+u_{t-1}\right] X_{2, t-1}+\eta_{2, t}
\end{aligned}\right.
$$

In this case, the diagram in Figure 1 of [33] can be simplified by the following diagram:

From the diagram in Figure 1, one can clearly see that causal mechanism from $u$ to $X_{2}$ for equation (1) is a process which involves an indirect causal effect and a direct causal effect. So, causal mechanism actually consists of direct and indirect causal effects. Hence, for a multivariate linear regression model with time-invariant coefficients, the influence from one variable to the other variable is always called causal effect or

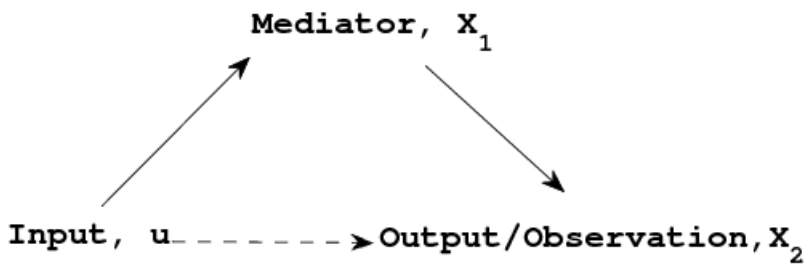

Fig. 1. Diagram of causal mechanism from $u$ to $X_{2}$ for equation (1): $\rightarrow$ represents the causal (indirect) effect of the input $u$ on the outcome $X_{2}$ which is transmitted through the intermediate variable or the mediator $X_{1}$; $\rightarrow$ represents causal (direct) effect of the input $u$ on the outcome $X_{2}$.

causal influence. ii) The authors in [32] said that NC is causal mechanism because it includes coefficients of the regression model. In fact, mathematically, GC in time domain and all Granger-alike causalities in frequency domain also include coefficients of the regression model. An immediate example is PDC which includes nothing except for coefficients of the regression model. However, in the literature, nobody says that GC in time domain and Granger-alike causalities in frequency domain discuss causal mechanism, but causal effect or causal influence. Therefore, in this way, NC also discusses causal effect or causal influence like GC and Granger-alike causalities. iii) The authors in [32] think that causal effect in the case of GC (time domain) mainly lies in the fact that adding $X_{2}$ can causes the reduction of prediction error of $X_{1}$. However, in the literature Granger-alike causality approaches (such as PDC, RPC, DTF) in frequency domain do not talk about "the reduction of prediction error" because these approaches use only the transfer function (based on joint regression model) and has nothing to do with auto regression model (so, there is no "the reduction of prediction error"). But, in the literature they are all called to reflect causal effect or causal influence, not causal mechanism.

In fact, GC, Hu et al's NC, and Granger-like causality tools are all to describe not only existence of causal effect or causal influence and but also the strength of causal effect or causal influence. To reveal the real strength of causal effect one must use the whole information from the model which of course includes the time-invariant coefficients. Using $\mathrm{NC}$ method, in [30] $\mathrm{Hu}$ et al showed that the causal effect changes as the time-invariant coefficients change (see, e.g., Fig.10(b) of [30]). Additionally, even in the nonlinear dynamical regression model (1) of [35], convergent cross mapping (CCM) was designed to describe the different causal effects as the timeinvariant coefficient parameters are different (see Fig.3B [35]). In summary, whether or not a causality method involving the time-invariant coefficients of the model is not the reason that this causality method reveals causal mechanism not causal effect!

In neuroscience, currently multi-channels of EEG recording and functional imaging play a more and more important role in studying network mechanisms for cognition and disease diagnosis. For example, we may be interested in causal flow among different subsets (or group, blocks) of the recorded brain areas. Since there may not exist causal interactions between two single time series but there may exist causality among blocks of time series, to reveal directional causal 
influence among different blocks, currently there are two standard approaches: one approach is to either average or extract a principal component to obtain a single time series from each block and then apply the bivariate GC [13], [36], [37]; the other one is to directly calculate the bivariate GC for all pairwise combinations where one time series is from the first block and the other one is from the second block and then summarize pairwise causality values as the total GC between the two blocks. Reasonability of the first approach is questionable because averaging or a principal component can not completely represent all data channels in one block. The second approach is intuitive but has the following shortcomings: 1) it is computationally cumbersome; 2) it may cause unexpected uncertainty because the estimated model may have different parameters (such as model order) for different blocks of data, as a result may lead to erroneous results in practice [38], [39]. To overcome these shortcomings, BGC and spectral BGC were proposed for multivariate time series [13], [40], [41]. Since BGC or spectral BGC is an extension of the bivariate GC to blockwise case and the bivariate GC may not disclose true causality between two univariate time series as pointed out in detail in [30], inevitably, they may not be able to disclose real causal flow among different blocks. Their shortcomings will be discussed in detail later in this paper.

It is noted that NC and spectral NC in [30] can disclose direct causality influence between any two single time series in a linear regression model. In this paper, on one hand, we demonstrate that (i) BGC in time domain may not disclose true causality. (ii) Spectral BGC and conditional spectral BGC have many shortcomings/limitations since these methods use the transfer function (or its inverse matrix) and partial information of the model, which may lead to misinterpretation results. On the other hand, we extend $\mathrm{NC}$ in time and frequency domains to blockwise case based on the Frobenius norm of a vector or matrix and propose BNC and spectral BNC. Finally, we demonstrate that the proposed BNC measures are advantageous over existing methods by several illustrative examples. In particular, for a real ERP EEG data from a seizure patient spectral BNC gives promising results while the spectral BGC generates misleading results. Thus, our proposed BNC measures may shed brand-new light on blockwise causality analysis. We envision that the proposed BNC measures will have a wide range of applications in other areas, such as economic science, engineering, as well as neuroscience.

We organize the paper as below. Section II introduces blockwise causality methods in time and frequency domains. Section III provides validation examples of blockwise causality analysis. Section IV makes concluding remarks.

\section{MEthodS}

\section{A. Blockwise Causality Analysis in Time Domain}

We will first introduce the existing $\mathrm{BGC}$ and conditional $\mathrm{BGC}$ in this section. We will then propose a BNC.

1) BGC and Conditional BGC: Suppose, given two blocks of time series $X_{i}=\left[x_{i 1}, \ldots, x_{i p_{i}}\right]^{\top}, i=1,2$, which are assumed to be jointly stationary where " $T$ " denotes the matrix transposition. $X_{1}$ and $X_{2}$ have the following autoregressive model

$$
\left\{\begin{array}{l}
X_{1, t}=\sum_{j=1}^{m} \mathbf{A}_{11, j} X_{1, t-j}+\epsilon_{1, t} \\
X_{2, t}=\sum_{j=1}^{m} \mathbf{A}_{22, j} X_{1, t-j}+\epsilon_{2, t}
\end{array}\right.
$$

and $X_{1}$ and $X_{2}$ have the following joint regression model

$$
\left\{\begin{array}{l}
X_{1, t}=\sum_{j=1}^{m} A_{11, j} X_{1, t-j}+\sum_{j=1}^{m} A_{12, j} X_{2, t-j}+\eta_{1, t} \\
X_{2, t}=\sum_{j=1}^{m} A_{21, j} X_{1, t-j}+\sum_{j=1}^{m} A_{22, j} X_{2, t-j}+\eta_{2, t}
\end{array}\right.
$$

where $t=0,1, \cdots, N, \mathbf{A}_{i i, j}$ is $p_{i} \times p_{i}$ dimensional matrix, $A_{i k, j}$ is a $p_{i} \times p_{k}$ dimensional matrix $(k=1,2), \epsilon_{i}=$ $\left[\epsilon_{i 1}, \ldots, \epsilon_{i p_{i}}\right]^{\top}$ and $\eta_{i}=\left[\eta_{i 1}, \ldots, \eta_{i p_{i}}\right]^{\top}$ are two random noise vectors, $\epsilon_{i j}$ and $\eta_{i j}$ have zero mean, $j=1, \cdots, p_{i}, i=$ 1,2 . The covariance matrix of $\epsilon_{1}$ and $\epsilon_{2}$ is given by $\Sigma_{1}=$ $E\left(\epsilon_{1} \epsilon_{1}^{\top}\right), \Gamma_{1}=E\left(\epsilon_{2} \epsilon_{2}^{\top}\right)$ respectively and the contemporaneous covariance matrix of $\eta_{1}$ and $\eta_{2}$ is

$$
\Sigma=E\left(\left(\begin{array}{l}
\eta_{1} \\
\eta_{2}
\end{array}\right)\left(\begin{array}{ll}
\eta_{1}^{\top} & \eta_{2}^{\top}
\end{array}\right)\right)=\left(\begin{array}{ll}
\Sigma_{2} & \Upsilon_{2} \\
\Upsilon_{2}^{\prime} & \Gamma_{2}
\end{array}\right)
$$

If we check the first two equalities in equation (2) and (3), the BGC ([36], [42]), from $X_{2}$ to $X_{1}$ is defined by

$$
F_{X_{2} \rightarrow X_{1}}=\ln \frac{\left|\Sigma_{1}\right|}{\left|\Sigma_{2}\right|}
$$

where $|\cdot|$ denotes the determinate of the enclosed matrix. Obviously, $F_{X_{2} \rightarrow X_{1}}=0$ if no causal flow from $X_{2}$ to $X_{1}$ exists and $F_{X_{2} \rightarrow X_{1}}>0$ if there is. The causal flow from $X_{1}$ to $X_{2}$ is similarly given by

$$
F_{X_{1} \rightarrow X_{2}}=\ln \frac{\left|\Gamma_{1}\right|}{\left|\Gamma_{2}\right|}
$$

Given two blocks of time series, there are two types of interactions between these two blocks: the interaction is direct or the interaction is mediated by another block of time series. In order to determine the type of interactions (direct or mediated), the conditional BGC ([13], [42]) was proposed as

$$
F_{X_{2} \rightarrow X_{1} \mid X_{3}}=\ln \frac{\left|\operatorname{var}\left(X_{1, t} \mid \tilde{X}_{1, t-1}, \tilde{X}_{3, t-1}\right)\right|}{\left|\operatorname{var}\left(X_{1, t} \mid \tilde{X}_{1, t-1}, \tilde{X}_{2, t-1}, \tilde{X}_{3, t-1}\right)\right|}
$$

where $\tilde{X}_{i, t-1}$ is denoted as the subspace of $\left\{X_{i, s} ; s \leq t-1, i=\right.$ $1,2,3\}, X_{3}=\left[x_{31}, \cdots, x_{3 p_{3}}\right]^{\top}, X_{1}, X_{2}, X_{3}$ are three blocks of time series of two blockwise regressive models below:

$$
X_{1, t}=\sum_{j=1}^{m} \mathbf{a}_{11, j} X_{1, t-j}+\sum_{j=1}^{m} \mathbf{a}_{13, j} X_{3, t-j}+\epsilon_{3, t}
$$

and $X_{1, t}=$

$$
\sum_{j=1}^{m} a_{11, j} X_{1, t-j}+\sum_{j=1}^{m} a_{12, j} X_{2, t-j}+\sum_{j=1}^{m} a_{13, j} X_{3, t-j}+\eta_{3, t} .
$$

where $\epsilon_{3}=\left[\epsilon_{i 1}, \ldots, \epsilon_{i p_{3}}\right]^{\top}$ and $\eta_{3}=\left[\eta_{i 1}, \ldots, \eta_{i p_{3}}\right]^{\top}$ are two random noise vectors.

In all illustrative examples in this paper, for simplicity we always assume $p_{i}=2$, that is, the block is a vector with two elements. 
In order to give a better explanation for $\mathrm{BGC}$, we would like to first introduce the properties of BGC as below:

Property 1: (i) Let's take the following model as an example,

$$
\left\{\begin{array}{l}
x_{11, t}=a_{13,1} x_{21, t-1}-0.1 x_{22, t-1}+\eta_{11, t} \\
x_{12, t}=-0.1 x_{21, t-1}-0.7 x_{22, t-1}+\eta_{12, t} \\
x_{21, t}=0.7 x_{21, t-1}+0.1 x_{22, t-1}+\eta_{21, t} \\
x_{22, t}=0.1 x_{21, t-1}+0.7 x_{22, t-1}+\eta_{22, t}
\end{array}\right.
$$

where $\eta_{i j}$ are four independent white noise processes with zero mean and variance of $\sigma_{\eta_{21}}^{2}=1=\sigma_{\eta_{22}}^{2}, i, j=1,2$. We demonstrate two different cases of BGC values in Fig. 2: (1) $a_{13,1}=-0.7$ and $\sigma_{\eta_{11}}^{2}=\sigma_{\eta_{12}}^{2}$ ranges from 0.01 to 2 at the same time; (2) $a_{13,1}$ ranges from -0.15 to -0.95 and $\sigma_{\eta_{11}}^{2}=$ $1=\sigma_{\eta_{12}}^{2}$. We can easily tell, from Fig. 2(a) and Fig. 2(b), that the $\mathrm{BGC}$ values derived from $X_{2}$ to $X_{1}$ will decrease if the variance value of $\sigma_{\eta_{11}}^{2}$ and $\sigma_{\eta_{12}}^{2}$ increases (or increases if the amplitude $\left|a_{13,1}\right|$ increases). This indicates that the increasing of the amplitude $\left|a_{13,1}\right|$ or the decreasing of variance of the residual term $\eta_{1}$ will cause the increasing of $\mathrm{BGC}$ value from $X_{2}$ to $X_{1}$. Therefore, we can draw a conclusion that the BGC value is actually a relative concept. If we find a larger BGC value from $X_{2}$ to $X_{1}$, this indicates that, compared to the influence from the residual term $\eta_{1, t}$, the causal influence from the first term $A_{12,1} X_{2, t-1}\left(A_{12,1}=\left[a_{i j, 1}\right]_{2 \times 2}, i=1,2, j=\right.$ $\left.3,4, a_{14,1}=-0.1, a_{23,1}=-0.1, a_{24,1}=-0.7\right)$ is occupying a larger portion, or vice versa. (a)

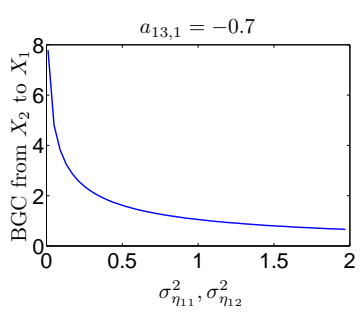

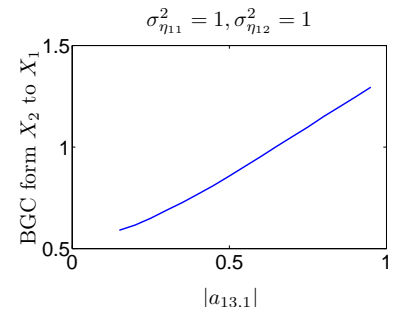

(b)
Fig. 2. (a) This figure shows the BGC values from $X_{2}$ to $X_{1}$ in (10) as variance $\sigma_{\eta_{11}}^{2}$ and $\sigma_{\eta_{12}}^{2}$ changes from 0.01 to 2 where $a_{13,1}=-0.7$. (b) This figure demonstrates the BGC values from $X_{2}$ to $X_{1}$ in (10) as $a_{13,1}$ changes from -0.15 to -0.95 where $\sigma_{\eta_{11}}^{2}=1=\sigma_{\eta_{12}}^{2}$. From Figure.(a), we can easily tell that the BGC value from $X_{2}$ to $X_{1}$ will decrease, if the variance of $\sigma_{\eta_{11}}^{2}$ or $\sigma_{\eta_{12}}^{2}$ increases. From Figure. (b), we can easily tell that the BGC value from $X_{2}$ to $X_{1}$ will increase, if the amplitude of $\left|a_{13,1}\right|$ increases.

(ii) Now, let's take a look at the model as below

$\left\{\begin{array}{l}x_{11, t}=a_{11,1} x_{11, t-1}+a_{12,1} x_{12, t-1}-0.2 x_{21, t-1}-0.1 x_{22, t-1}+\eta_{11, t} \\ x_{12, t}=a_{21,1} x_{11, t-1}+a_{22,1} x_{12, t-1}-0.1 x_{21, t-1}-0.1 x_{22, t-1}+\eta_{12, t} \\ x_{21, t}=a_{31,1} x_{11, t-1}+a_{32,1} x_{12, t-1}+0.2 x_{21, t-1}+0.1 x_{22, t-1}+\eta_{21, t} \\ x_{22, t}=a_{41,1} x_{11, t-1}+a_{42,1} x_{12, t-1}+0.1 x_{21, t-1}+0.1 x_{22, t-1}+\eta_{22, t}\end{array}\right.$

where $0<a_{i k, 1}, a_{j k, 1}<1$ and for simplicity, we will assume that $\eta_{i k}$ are four independent white noise processes with zero mean and variance of $\sigma_{\eta_{11}}^{2}=1=\sigma_{\eta_{12}}^{2}, \sigma_{\eta_{21}}^{2}=1=\sigma_{\eta_{22}}^{2}$, $i=1,2, j=3,4, k=1$, 2. Fig. 3 shows BGC from $X_{2}$ to $X_{1}$ for (11) under different parameters $a_{i k, 1}$ and $a_{j k, 1}$. One thing we would like to point out is that, when we compute the BGC value, for each specific (11), we always generate a data set of 200 realizations of 10000 time points and then estimate autoregressive representation model (2) and joint representations model (3), with the order of 8 , via employing the least-squares method and calculating the BGC value where the order 8 fits for every examples in this section [please refer to Fig. 3(a) and (b) from which we can easily tell that BGC values are steady when the order of the estimated models is larger than 8]. Next, we calculate the average value across all realizations and obtain the BGC value from $X_{2}$ to $X_{1}$. From Fig. 3, we can clearly find out that the BGC value from $X_{2}$ to $X_{1}$ is not related to parameters $a_{i k, 1}$ and $a_{j k, 1}$ [parameters $a_{i k, 1}$ and $a_{j k, 1}$ are chosen to make sure (11) is not diverge]. It should be noted that this result is consistent with spectral $\mathrm{BGC}$ result introduced later in this paper.

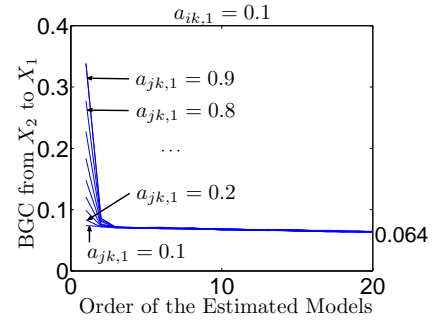

(a)

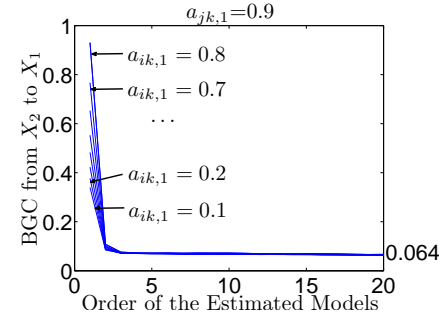

(b)
Fig. 3. (a) This figure shows the BGC value from $X_{2}$ to $X_{1}$ is a function of the order of the estimated model for Model (11) when $a_{i k, 1}=0.1$ and $a_{j k, 1}$ is changed from 0.1 to 0.9 . (b) This figure shows that the BGC value from $X_{2}$ to $X_{1}$ is a function of the order of the estimated model for (11) when $a_{j k, 1}=0.9$ and $a_{i k, 1}$ is changed from value 0.1 to value 0.8 . From Figures (a) and (b), we can easily tell that: (i) the BGC value from $X_{2}$ to $X_{1}$ is stable and converges to 0.064 when the order $p>8$; (ii) the BGC value from $X_{2}$ to $X_{1}$ is not related to the parameters $a_{i k, 1}$ and $a_{j k, 1}$.

(iii) For (3), if $\eta_{11, t}=\eta_{21, t}$ and $\eta_{12, t}=\eta_{22, t}$ at the same time or $\eta_{21, t} \equiv 0 \equiv \eta_{22, t}$, then BGC from $X_{2}$ to $X_{1}$ equals to zero. This can be proved from the spectral BGC (28) demonstrated later.

Generally speaking, in theory, the BGC value and the conditional BGC value (except the two specific cases in (iii) of Property 1 are very useful to determine if there is directional interaction between two blocks of time series or among three blocks of time series. However, a question is raised when the causal influence exists: will the BGC value or the conditional BGC value reflect the true causality? In order to answer this question, we will use the simple model below as an example for discussion.

$$
\left\{\begin{array}{l}
X_{1, t}=A_{12,1} X_{2, t-1}+\eta_{1, t} \\
X_{2, t}=A_{21,1} X_{1, t-1}+\eta_{2, t}
\end{array}\right.
$$

where $\eta_{1}=\left[\eta_{11}, \eta_{12}\right]^{\top}$ and $\eta_{2}=\left[\eta_{21}, \eta_{22}\right]^{\top}$ are two independent random vectors with zero mean and $A_{12,1} A_{21,1} \neq 0$. From (12) one can get

$$
\begin{aligned}
X_{1, t} & =A_{12,1}(\overbrace{A_{21,1} X_{1, t-2}+\eta_{2, t-1}}^{X_{2}, t-1})+\eta_{1, t} \\
& =A_{12,1} A_{21,1} X_{1, t-2}+A_{12,1} \eta_{2, t-1}+\eta_{1, t} .
\end{aligned}
$$

Thus, the BGC value $F_{X_{2} \rightarrow X_{1}}=$

$\ln \frac{\left|E\left(\left(A_{12,1} \eta_{2, t-1}+\eta_{1, t}\right)\left(A_{12,1} \eta_{2, t-1}+\eta_{1, t}\right)^{\top}\right)\right|}{\left|E\left(\eta_{1, t} \eta_{1, t}{ }^{\top}\right)\right|} \in[0,+\infty)$ 
from which one can see that (14) only involves two noise terms and does not include the first term of (13). However, all three terms make contributions to current $X_{1, t}$, and $A_{12,1} A_{21,1} X_{1, t-2}$ should have causal flow on $X_{1, t}$ and should be considered to demonstrate true causality from $X_{2}$ to $X_{1}$. In particular, if the covariance matrix of $\eta_{2}$ is equal to zeros, we obtain $X_{1, t}=A_{12,1} A_{21,1} X_{1, t-2}+\eta_{1, t}$, thus $F_{X_{2} \rightarrow X_{1}}=0$. Note that $A_{12,1} A_{21,1} \neq 0$ and $A_{21,1} X_{1, t-2}$ is from $X_{2, t-1}$, we see that $X_{2}$ has nonzero true causality on $X_{1}$. Therefore, this simple illustrative example demonstrates that BGC and conditional BGC methods may not necessarily disclose true causality. We summarize our comments in the following remark.

Remark 1. (i) If causality from $X_{2}$ to $X_{1}$ exists, $F_{X_{2} \rightarrow X_{1}}$ varies in $(0,+\infty)$. However, as shown above, the $\mathrm{BGC}$ value may not reflect true causality correctly. In other words, the BGC value may have nothing to do with true causality. Therefore, based on the the value of $F_{X_{2} \rightarrow X_{1}}$, it will be very difficult to conclude how much real impact or influence is caused. So, even there is a smaller value of $F_{X_{2} \rightarrow X_{1}}$ (e.g., it equals to 0.1 ), it does not necessarily indicate that $X_{2}$ has smaller (less) causal influence on $X_{1}$. On the other hand, when we obtain a larger value of $F_{X_{2} \rightarrow X_{1}}$ (e.g., it equals to 1) which can be ignored compared to $F_{X_{2} \rightarrow X_{1}}=+\infty$, we can not claim that there is strong causality from $X_{2}$ to $X_{1}$. Next we will show this by two illustrative models below:

$\left\{\begin{array}{l}x_{11, t}=0.7 x_{11, t-1}+0.2 x_{12, t-1}-0.2 x_{21, t-1}-0.1 x_{22, t-1}+\eta_{11, t} \\ x_{12, t}=0.2 x_{11, t-1}+0.7 x_{12, t-1}-0.1 x_{21, t-1}-0.1 x_{22, t-1}+\eta_{12, t} \\ x_{21, t}=0.2 x_{21, t-1}+0.1 x_{22, t-1}+\eta_{21, t} \\ x_{22, t}=0.1 x_{21, t-1}+0.1 x_{22, t-1}+\eta_{22, t}\end{array}\right.$

and

$$
\left\{\begin{array}{l}
x_{11, t}=-0.2 x_{21, t-1}-0.1 x_{22, t-1}+\eta_{31, t} \\
x_{12, t}=-0.1 x_{21, t-1}-0.1 x_{22, t-1}+\eta_{32, t} \\
x_{21, t}=0.2 x_{21, t-1}+0.1 x_{22, t-1}+\eta_{21, t} \\
x_{22, t}=0.1 x_{21, t-1}+0.1 x_{22, t-1}+\eta_{22, t}
\end{array}\right.
$$

where $\eta_{i j}$ are six independent white noise processes with zero mean and variances $\sigma_{\eta_{11}}^{2}=0.005=\sigma_{\eta_{12}}^{2}, \sigma_{\eta_{21}}^{2}=1=$ $\sigma_{\eta_{22}}^{2}, \sigma_{\eta_{31}}^{2}=0.05=\sigma_{\eta_{32}}^{2}, i=1,2,3, j=1,2$. For (15) we can obtain BGC $F_{X_{2} \rightarrow X_{1}}=2.94$. For (16) we can obtain BGC $F_{X_{2} \rightarrow X_{1}}=0.91$. Note that $\sigma_{\eta_{11}}^{2}$ and $\sigma_{\eta_{12}}^{2}$ of the noise terms $\eta_{11}, \eta_{12}$ in equation (15) are smaller. Therefore, a little or minor change (compared to $\sigma_{\eta_{11}}^{2}$ and $\sigma_{\eta_{12}}^{2}$ ) of the covariance of $\epsilon_{1}$ of the estimated autoregressive model, may result in a larger (or bigger) BGC value (see BGC definition in (5) for more detail), as shown in $F_{X_{2} \rightarrow X_{1}}=2.94$. We can conduct similar analysis for (16). Hence, we can conclude that both BGC values are "intuitively reasonable" according to BGC defintion. Now, we have to raise one question: will the "reasonable" BGC values better reflect the true causality or trend of the true causality? Unfortunately, the answer to this question is "No". There are four observations we should note. First, $X_{2}$ is same in both (15) and (16); secondly, $X_{1}$ is driven by $X_{2}$ in (16); thirdly, $X_{1}$ is driven by $X_{1}$ and $X_{2}$ in (15); and fourthly, impact (influence) from $\eta_{1}$ or $\eta_{3}$ is very small and it can even be ignored since their covariances are very small. As a result, we can conclude, intuitively, that the true causality from $X_{2}$ to $X_{1}$ in (15) should be weaker than that in (16). However, we should note that $F_{X_{2} \rightarrow X_{1}}=2.94$ for (15) is larger than $F_{X_{2} \rightarrow X_{1}}=0.91$ for (16). Therefore, the $\mathrm{BGC}$ value is unbelievable for at least in the aforementioned two cases. In other words, the resulting BGC value won't be true to reflect the true causality. So, generally speaking, we can conclude that BGC value may not necessarily reflect true causal influence between blocks of time series.

(ii) For conditional BGC, the same problem mentioned in (i) exists.

(iii) Similar to (iii) of Remark 1 [30], in the case of blockwise, the indirect Granger causality along with a route: $X_{2} \rightarrow X_{3} \rightarrow X_{1}$ does not satisfy the cascade property, that is, $F_{X_{2} \rightarrow X_{1}} \neq F_{X_{2} \rightarrow X_{3}} \times F_{X_{3} \rightarrow X_{1}}$.

2) $B N C$ : Note the above mentioned shortcomings/limitations of BGC, we next give the BNC definition for multivariate stationary processes. Use the following general model:

$\left\{\begin{array}{c}X_{1, t}=\sum_{j=1}^{m} A_{11, j} X_{1, t-j}+\cdots+\sum_{j=1}^{m} A_{1 n, j} X_{n, t-j}+\eta_{1, t} \\ X_{2, t}=\sum_{j=1}^{m} A_{21, j} X_{1, t-j}+\cdots+\sum_{j=1}^{m} A_{2 n, j} X_{n, t-j}+\eta_{2, t} \\ \vdots \\ X_{n, t}=\sum_{j=1}^{m} A_{n 1, j} X_{1, t-j}+\cdots+\sum_{j=1}^{m} A_{n n, j} X_{n, t-j}+\eta_{n, t}\end{array}\right.$

where $t=0,1, \ldots, N, X_{i}=\left[x_{i 1}, \ldots, x_{i p_{i}}\right]^{\top}$ are multivariate stationary processes, $\eta_{i}=\left[\eta_{i 1}, \ldots, \eta_{i p_{i}}\right]^{\top}$ is a noise vector with $\eta_{i j}$ having zero mean, $j=1, \cdots, p_{i}$. $A_{k i}$ is a $p_{k} \times p_{i}$ dimensional matrix, $k, i=1, \ldots, n$. From equation (17), we can easily tell that Fig. 4 completely describes all contributions to $X_{k, t}$. The contributions includes $\sum_{j=1}^{m} A_{k 1, j} X_{1, t-j}, \ldots, \sum_{j=1}^{m} A_{k n, j} X_{n, t-j}$ and the noise $\eta_{k, t}$ where the contribution of $\sum_{j=1}^{m} A_{k k, j} X_{k, t-j}$ is causality influence from $X_{k}$ 's previous values. In other words, each contribution in Fig. 4 should be included in order to determine $X_{k, t}$. When we consider proportions of these contributions, the following is an obvious fact: If $\sum_{j=1}^{m} A_{k i, j} X_{i, t-j}$ occupies larger portion, then $X_{i}$ has stronger true causality on $X_{k}$. Based on this fact, any ideal causality definition from $X_{i}$ to $X_{k}$ should better describe what proportion $X_{i}$ has among all these contributions. Obviously this is a general guideline to define

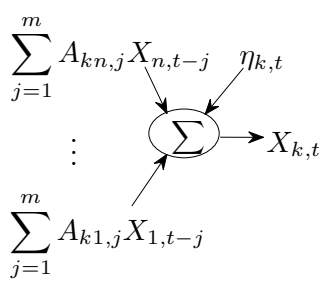

Fig. 4. This figure shows the contributions to $X_{k, t}$

any causality tool based on linear regression model (i.e., one must consider all contributions to the variable). Now, we will 
define a new direct blockwise causality from $X_{i}$ to $X_{k}$ below:

$$
N_{X_{i} \rightarrow X_{k}}=\frac{\sum_{t=m}^{N}\left\|\sum_{j=1}^{m} A_{k i, j} X_{i, t-j}\right\|_{F}^{2}}{\sum_{h=1}^{n} \sum_{t=m}^{N}\left\|\sum_{j=1}^{m} A_{k h, j} X_{h, t-j}\right\|_{F}^{2}+\sum_{t=m}^{N}\left\|\eta_{k, t}\right\|_{F}^{2}} .
$$

where $\|\cdot\|_{F}$ denotes the F-norm of the enclosed matrix, and when $N$ is enough large,

$$
\begin{aligned}
\sum_{t=m}^{N}\left\|\eta_{k, t}\right\|_{F}^{2} & =\sum_{t=1}^{N}\left\|\eta_{k, t}\right\|_{F}^{2}-\sum_{t=1}^{m}\left\|\eta_{k, t}\right\|_{F}^{2} \\
& =N \sum_{l=1}^{p_{k}} \sigma_{\eta_{k l}}^{2}-\sum_{t=1}^{m}\left\|\eta_{k, t}\right\|_{F}^{2} \\
& \approx N \sum_{l=1}^{p_{k}} \sigma_{\eta_{k l}}^{2} .
\end{aligned}
$$

Then, (18) can be approximated as

$$
N_{X_{i} \rightarrow X_{k}}=\frac{\sum_{t=m}^{N}\left\|\sum_{j=1}^{m} A_{k i, j} X_{i, t-j}\right\|_{F}^{2}}{\sum_{h=1}^{n} \sum_{t=m}^{N}\left\|\sum_{j=1}^{m} A_{k h, j} X_{h, t-j}\right\|_{F}^{2}+N \sum_{l=1}^{p_{k}} \sigma_{\eta_{k l}}^{2}} .
$$

In this paper, we always suppose that $N$ is large enough so that $N_{X_{i} \rightarrow X_{X_{k}}}$ can be described as (20). Generally speaking, we will be able to make some important comments as follows.

Remark 2: (i) For (15) and (16), we can get $N_{X_{2}} \underset{\rightarrow}{\rightarrow} X_{1}=$

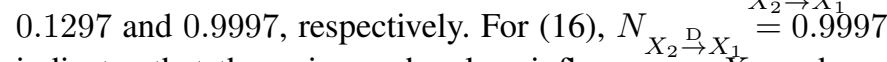
indicates that the noise $\eta_{3}$ has less influence on $X_{1}$ and can be ignored. So the true causality from $X_{2}$ to $X_{1}$ for (16) is close to that of the following model

$$
\left\{\begin{array}{l}
x_{11, t}=-0.2 x_{21, t-1}-0.1 x_{22, t-1} \\
x_{12, t}=-0.1 x_{21, t-1}-0.1 x_{22, t-1} \\
x_{21, t}=0.2 x_{21, t-1}+0.1 x_{22, t-1}+\eta_{21, t} \\
x_{22, t}=0.1 x_{21, t-1}+0.1 x_{22, t-1}+\eta_{22, t}
\end{array}\right.
$$

whose true causality from $X_{2}$ to $X_{1}$ obviously equals to 1 (that is, the strongest causality). Note the noise terms $\eta_{1 i}$ in (15) have small variances, one can see that the true causality from $X_{2}$ to $X_{1}$ for (15) is close to that of the following model

$$
\left\{\begin{array}{l}
x_{11, t}=0.7 x_{11, t-1}+0.2 x_{12, t-1}-0.2 x_{21, t-1}-0.1 x_{22, t-1} \\
x_{12, t}=0.2 x_{11, t-1}+0.7 x_{12, t-1}-0.1 x_{21, t-1}-0.1 x_{22, t-1} \\
x_{21, t}=0.2 x_{21, t-1}+0.1 x_{22, t-1}+\eta_{21, t} \\
x_{22, t}=0.1 x_{21, t-1}+0.1 x_{22, t-1}+\eta_{22, t}
\end{array}\right.
$$

Since $X_{1}$ 's past value makes contribution to $X_{1}$ 's current value in (22), we can easily tell that the true causality from $X_{2}$ to $X_{1}$ for (22) is definitely weaker than that for (21). For (15), the BNC value $N_{X_{2}} \stackrel{\mathrm{D}}{\rightarrow} X_{1}=0.1297$ is indeed less than the BNC value $N_{X_{2}} \stackrel{\mathrm{D}}{\rightarrow} X_{1} \stackrel{\rightarrow}{=} 0.9997$ for (16). However, BGC value $F_{X_{2} \rightarrow X_{1}}=2.94$ for (15) is no less or equal to BGC value $F_{X_{2} \rightarrow X_{1}}=0.91$ for (16). This phenomena violates the aforementioned analysis. Thus, from these two examples BNC method defined in (18) is more sensitive to reflect true causality or trend of true causality than BGC method. (ii) When we consider the two specific cases in (iii) of Property 1 where $A_{12, j} \neq 0$, we have $F_{X_{2} \rightarrow X_{1}}=0$ for (3). But, one may easily find out that $N_{X_{2}} \stackrel{\mathrm{D}}{\rightarrow} X_{1} \neq 0$ if $X_{2} \not \equiv 0$, that is, there is true causality. Therefore, even if BGC $F_{X_{2} \rightarrow X_{1}}=0$, true causality may still exist. However, if BNC $N_{X_{2}} \stackrel{\mathrm{D}}{\rightarrow} X_{1}=0$, there must exist no true causality (no $\mathrm{BGC}$, of course). Therefore, BNC may reveal the strength of true causality more correctly than BGC.

(iii) For BNC method, we can check that the indirect causality from $X_{i}$ to $X_{k}$ via $X_{l}$ satisfies

$$
N_{X_{i}}{\stackrel{\text { ID }}{\rightarrow} X_{k} \text { via } X_{l}}=N_{X_{i} \stackrel{\mathrm{D}}{\rightarrow} X_{l}} \times N_{X_{l} \stackrel{\mathrm{D}}{\rightarrow} X_{k}} .
$$

This cascade property does not hold for the BGC method as discussed in (iii) of Remark 1.

(iv) Suppose we have a route $R: X_{i} \rightarrow X_{l_{1}}^{\prime} \rightarrow X_{l_{2}}^{\prime} \rightarrow$ $\cdots \rightarrow X_{l_{h}}^{\prime} \rightarrow X_{k}$ where $\left\{X_{l_{1}}^{\prime}, \ldots, X_{l_{h}}^{\prime}\right\} \subseteq\left\{X_{1}, \ldots, X_{n}\right\}-$ $\left\{X_{i}, X_{k}\right\}$, we can calculate the indirect causality from $X_{i}$ to $X_{k}$ via the route $R$ as follow: $N_{X_{i}} \stackrel{\text { ID }}{\rightarrow} X_{k}$ via route $R$

$$
=N_{X_{i} \stackrel{\mathrm{D}}{\rightarrow} X_{l_{1}}^{\prime}} \times \prod_{s=1}^{h-1} N_{X_{l_{s}}^{\prime} \stackrel{\mathrm{D}}{\rightarrow} X_{l_{s+1}^{\prime}}} \times N_{X_{l_{h}}^{\prime} \stackrel{\mathrm{D}}{\rightarrow} X_{k}} .
$$

v) It is easy to see that direct BNC in (20) is a natural extension of direct $\mathrm{NC}$ (20) in [30] to blockwise multivariate case.

Given a model, generally speaking, we don't know exactly what the true causality is. However, we have pointed out (from (i) and (iii) of Remark 2) that BNC method is more sensitive to reveal the true causality or the trend of the true causality than BGC method which may generate wrong result.

\section{B. Blockwise Causality Analysis in Frequency Domain}

In the previous section, we discussed the blockwise causality in time domain. We will introduce the blockwise causality in frequency domain in this section. We first propose a new causality definition called spectral BNC. Then we describe traditional spectral BGC and conditional spectral BGC measures and also point out their shortcomings/limitations.

1) Spectral BNC : Let's use the general model (17) as an example. We will take Fourier transformation on both side of equation (17) and obtain

$\left\{\begin{array}{l}X_{1}(f)=A_{11}(f) X_{1}(f)+\cdots+A_{1 n}(f) X_{n}(f)+\eta_{1}(f) \\ X_{2}(f)=A_{21}(f) X_{1}(f)+\cdots+A_{2 n}(f) X_{n}(f)+\eta_{2}(f) \\ \vdots \\ X_{n}(f)=A_{n 1}(f) X_{1}(f)+\cdots+A_{n n}(f) X_{n}(f)+\eta_{n}(f)\end{array}\right.$

where

$$
A_{l j}(f)=\sum_{k=1}^{m} A_{l j, k} e^{-i 2 \pi f k}, i=\sqrt{-1}, l, j=1, \ldots, n .
$$

From (24), we can tell that contributions to $X_{k}(f)$ involve $n$ parts: $A_{k i}(f) X_{i}(f),(i=1,2, \cdots, n i \neq k)$, the noise term $\eta_{k}(f)$, and $A_{k k}(f) X_{k}(f)$ which constitutes contribution from $X_{k}$ 's past values. See Fig. 5 for more clearness. Based on these contributions we can naturally propose a blockwise new 


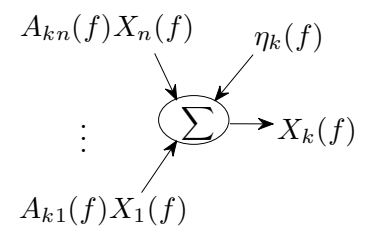

Fig. 5. This figure shows all contributions to $X_{k}(f)$.

direct causality from $X_{i}$ to $X_{k}$ in frequency domain (called by spectral BNC) as below: $N_{X_{i} \stackrel{\mathrm{D}}{\rightarrow}_{X_{k}}}(f)=$

$$
\frac{\left\|A_{k i}(f)\right\|_{F}^{2}\left\|S_{X_{i} X_{i}}(f)\right\|_{F}}{\sum_{h=1}^{n}\left\|A_{k h}(f)\right\|_{F}^{2}\left\|S_{X_{h} X_{h}}(f)\right\|_{F}+\sum_{j=1}^{p_{k}} \sigma_{\eta_{k j}}^{2}},
$$

$i, k=1, \ldots, n, i \neq k$, and the spectrum matrix of $X_{i}=$ $\left[x_{i 1}, \ldots, x_{i p_{i}}\right]^{\mathrm{T}}$ is

$S_{X_{i} X_{i}}(f)=\left(\begin{array}{cccc}S_{x_{i 1} x_{i 1}}(f) & S_{x_{i 1} x_{i 2}}(f) & \cdots & S_{x_{i 1} x_{i p_{i}}}(f) \\ S_{x_{i 2} x_{i 1}}(f) & S_{x_{i 2} x_{i 2}}(f) & \cdots & S_{x_{i 2} x_{i p_{i}}}(f) \\ \vdots & \vdots & \ddots & \vdots \\ S_{x_{i p_{i}} x_{i 1}}(f) & S_{x_{i p_{i}} x_{i 2}}(f) & \cdots & S_{x_{i p_{i}} x_{i p_{i}}}(f)\end{array}\right)$,

where $S_{x_{i j} x_{i j}}(f)$ is the spectrum of $x_{i j}, S_{x_{i l} x_{i h}}(f)$ denotes the cross power spectral of $x_{i l}$ and $x_{i h} . j, l, h=1, \ldots, p_{i}, l \neq h$.

Remark 3: (i) We can easily find out that $0 \leq$ $N_{X_{i}{ }_{\rightarrow}^{\mathrm{D}} X_{k}}(f) \leq 1 . N_{X_{i}{ }_{\rightarrow}^{\mathrm{D}} X_{k}}(f) \equiv 0 \Longleftrightarrow A_{k i}(f) \equiv 0$, that is, all the coefficient matrices $A_{k i, 1}, \ldots, A_{k i, m}$ are zero matrices. $N_{X_{i} \stackrel{\mathrm{D}}{\rightarrow} X_{k}}(f) \equiv 1$ is equivalent to that $\eta_{k}$ is a zero vector and $A_{k j}(f) \equiv 0, j=1, \ldots, n, j \neq i$, that is, all the coefficient matrices $A_{k j, 1}, \ldots, A_{k j, m}$ are zeros, $j=1, \ldots, n, j \neq i$, i.e., the $k$ th equality in (24) can be expressed as

$$
X_{k, t}=\sum_{j=1}^{m} A_{k i, j} X_{i, t-j}
$$

which means $X_{k}$ is completely driven by $X_{i}$ 's past values.

(ii) We can define the indirect causality from $X_{i}$ to $X_{k}$ via $X_{l}$ as

$$
N_{X_{i} \stackrel{\text { ID }}{\rightarrow} X_{k} \text { via } X_{l}}(f)=N_{X_{i} \stackrel{\mathrm{D}}{\rightarrow} X_{l}}(f) \times N_{X_{l} \stackrel{\mathrm{D}}{\rightarrow} X_{k}}(f) .
$$

(iii) Suppose we have a route $R: X_{i} \rightarrow X_{l_{1}}^{\prime} \rightarrow X_{l_{2}}^{\prime} \rightarrow$ $\cdots \rightarrow X_{l_{h}}^{\prime} \rightarrow X_{k}$ where $\left\{X_{l_{1}}^{\prime}, \ldots, X_{l_{h}}^{\prime}\right\} \subseteq\left\{X_{1}, \ldots, X_{n}\right\}-$ $\left\{X_{i}, X_{k}\right\}$, the indirect causality from $X_{i}$ to $X_{k}$ via this route $R$ can be defined as $N_{X_{i}} \stackrel{\text { ID }}{\rightarrow} X_{k}$ via route $R$

$$
=N_{X_{i} \mathrm{D}_{X_{l_{1}}^{\prime}}}(f) \times \prod_{s=1}^{h-1} I_{X_{i_{s}}^{\prime}{ }^{\mathrm{D}} X_{l_{s+1}^{\prime}}}(f) \times N_{X_{l_{h}} \stackrel{\mathrm{D}}{\rightarrow} X_{k}}(f) .
$$

v) One can easily see that direct spectral BNC in (26) is a natural extension of spectral direct new causality (30) in [30] to blockwise multivariate case.

2) Spectral BGC and Conditional Spectral BGC: In the literature, many spectral causality measures have been proposed. We next only focus on the widely used spectral BGC and conditional spectral BGC and point out their shortcomings/limitations.
Spectral BGC ([40]-[42]): Given two blocks of time series (3), the spectral $\mathrm{BGC}$ from $X_{2}$ to $X_{1}$ is given by $F_{X_{2} \rightarrow X_{1}}(f)=$

$$
\ln \frac{\left|H_{11}(f) \Sigma_{2} H_{11}^{*}(f)+H_{12}(f) \tilde{\Gamma}_{2} H_{12}^{*}(f)\right|}{\left|H_{11}(f) \Sigma_{2} H_{11}^{*}(f)\right|} \in[0,+\infty)
$$

where the symbol " $*$ " denotes complex conjugate matrix transposition, the transfer function is given as $H(f)=\tilde{B}^{-1}(f) \triangleq$ $\left[H_{i j}(f)\right]_{2 \times 2}$ where

$H_{11}(f)=\tilde{B}_{22}(f) / \operatorname{det}(\tilde{B}), H_{12}(f)=-\tilde{B}_{12}(f) / \operatorname{det}(\tilde{B})$, $H_{21}(f)=-\tilde{B}_{21}(f) / \operatorname{det}(\tilde{B}), H_{22}(f)=\tilde{B}_{11}(f) / \operatorname{det}(\tilde{B})$,

$\tilde{B}=\left[\tilde{B}_{i j}(f)\right]_{2 \times 2}, \quad \tilde{B}_{1 l}(f)=B_{1 l}(f), \quad \tilde{B}_{2 l}(f)=B_{2 l}(f)-$ $\Upsilon_{2}^{\prime} \Sigma_{2}{ }^{-1} B_{1 l}(f), \quad B=\left[B_{i j}(f)\right]_{2 \times 2}, \quad B_{k k}(f)=I-$ $\sum_{j=1}^{m} A_{k k, j} e^{-i 2 \pi f j}, B_{h l}(f)=-\sum_{j=1}^{m} A_{h l, j} e^{-i 2 \pi f j}, \tilde{\Gamma}_{2}=\Gamma_{2}-$ $\Upsilon_{2}^{\prime} \Sigma_{2}{ }^{-1} \Upsilon_{2}, l, h, k=1,2, h \neq l$.

Shortcomings/limitations of the spectral BGC are pointed out in the following remark.

Remark 4: (i) There are same problems as in (i) and (iii) of Remark 1 for the spectral BGC method.

(ii) As shown in the two specific cases in (iii) of Property 1 and for any coefficient matrices $A_{11, j}, A_{12, j}, A_{21, j}, A_{22, j}$, we can calculate $F_{X_{2} \rightarrow X_{1}}(f) \equiv 0$ from (28). But, we can check $N_{X_{2}{ }_{\rightarrow} X_{1}}(f) \neq 0$ for (3) if $A_{12}(f) \neq 0$ and $X_{2}(f) \neq 0$, this means, the real causality exists. In fact, the true causality indeed exists by observing the model in any of these two cases. Therefore, the spectral BNC method can reveal nonzero true causality even in the case of BGC value being zero.

(iii) From (28) and (29) above, we can derive $F_{X_{2} \rightarrow X_{1}}(f)=$

$$
\ln \frac{\left|\bar{H}_{11}(f) \Sigma_{2} \bar{H}_{11}^{*}(f)+\bar{H}_{12}(f) \tilde{\Gamma}_{2} \bar{H}_{12}^{*}(f)\right|}{\left|\bar{H}_{11}(f) \Sigma_{2} \bar{H}_{11}^{*}(f)\right|}
$$

where $\bar{H}_{11}(f)=B_{22}(f)-\Upsilon_{2}^{\prime} \Sigma_{2}^{-1} B_{12}(f), \bar{H}_{12}(f)=B_{12}(f)$, we can easily tell that $F_{X_{2} \rightarrow X_{1}}(f)$ has nothing to do with $A_{11}(f)$ and $A_{21}(f)$, or, the true casuality is not related to the coefficient matrices $A_{11, j}$ and $A_{21, j}$. This may not be correct [please refer to the similar discussion in (i) of Remark 2 for more details].

(iv) The spectral conditional BGC [41] (omitted here for space reason) has the same problem as introduced in (ii) above.

In conclusion, for the spectral BGC and conditional spectral BGC, we have clearly pointed out that they have inherent shortcomings/limitations. The main reason lies in two aspects: i) these spectral BGC methods use the transfer function $H(f)$ or its inverse matrix whose diagonal element $\left(I-A_{i i}(f)\right)$ is totally different from the coefficient matrix $A_{i i}(f), i=$ $1, \cdots, n$; ii) as pointed in (iii) of Remark 4 , BGC measures only employ partial information and some information are missing. However, spectral BNC considers all contributions to $X_{k}(f)$ ) (see Fig. 5). Thus, the spectral BNC defined in (26) is mathematically more reasonable and understandable than spectral BGC methods.

\section{VALIDATION: FOUR EXAMPLES}

In this section, we will introduce four examples to demonstrate the effectiveness and advance of our proposed spectral BNC. We will first make comparison between spectral 
BNC and BGC measures using the first two examples in which we assume all noise terms are normal distribution and mutually independent (with variances of 1 in Example 1 and 0.3 in Example 2). We especially demonstrate some shortcomings/limitations of the existing BGC measures. In Example 3 we will discuss the synthetic simulation example of two univariate time series in simulation section [43] where even-related causality between two channels are known, and show that spectral NC or BNC is much better than spectral GC or BGC to reveal the true causality. In Example 4 (i.e., the last example 5 in [30]), we will then investigate eventrelated causality based on blocks of intracranial EEG from a patient suffering from seizure in left temporal lobe. The experiment includes recognizing pictures viewed (see [30] for more detail). We will compute the spectral BNC and spectral BGC for two blocks of EEGs: the first block involving two EEG channels is from left temporal lobe, and the second block involving two EEG channels is from right temporal lobe. Our simulation results demonstrates that the direct causal information influence from right side to left side can been clearly found by all blockwise measures. However, the spectral $\mathrm{BNC}$ result is consistent with the event-related activity and the spectral BGC result is not.

Example 1: In this example, we will make a comparison between spectral BGC and spectral BNC. Let's consider the following model:

$\left\{\begin{array}{l}x_{11, t}=a_{11,1} x_{11, t-1}+a_{12,1} x_{12, t-1}-0.6 x_{21, t-1}-0.6 x_{22, t-1}+\eta_{11, t} \\ x_{12, t}=a_{21,1} x_{11, t-1}+a_{22,1} x_{12, t-1}-0.3 x_{21, t-1}-0.3 x_{22, t-1}+\eta_{12, t} \\ x_{21, t}=0.6 x_{21, t-1}+0.6 x_{22, t-1}+\eta_{21, t} \\ x_{22, t}=0.3 x_{21, t-1}+0.3 x_{22, t-1}+\eta_{22, t}\end{array}\right.$

Let's consider two cases: $a_{i j, 1}=0.1$ and $a_{i j, 1}=0.5$ $(i=1,2 ; j=1,2)$. Intuitively, in both cases, the real causal influence from $X_{2}$ to $X_{1}$ should be completely different. The spectral BNC results in these two cases are reported in Fig. 6(b) from which one can see the different causal flows. However, based on (28), $X_{2}$ has the same spectral BGC on $X_{1}$ in these two cases [see Fig. 6(a)] because (28) has nothing to do with the coefficient $a_{i j, 1}$. Hence, we confirm that, generally speaking, spectral BGC may not be the true causal flow between two blocks of time series and may lead to wrong interpretation.

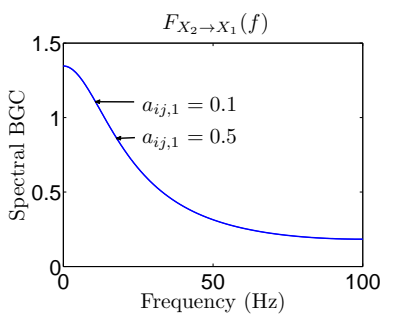

(a)

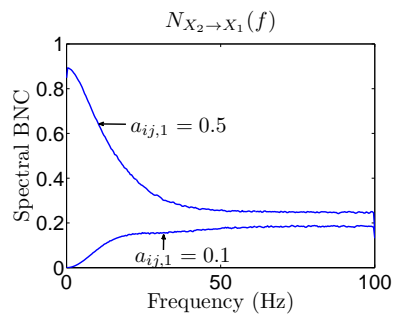

(b)
Fig. 6. (a) Spectral BGC results: $F_{X_{2} \rightarrow X_{1}}(f)$ based on (28) for (31). (b) Spectral BNC results: $N_{X_{2} \rightarrow X_{1}}(f)$ based on (26) for (31). In (a) and (b) two cases: $a_{i j, 1}=0.1$ and $a_{i j, 1}=0.5$, are considered.

Example 2: In this example, we will make a comparison between spectral BNC and conditional spectral BGC. Let's analyze the following model with six variables:

$$
\left\{\begin{aligned}
x_{11, t}= & -0.2 x_{11, t-1}+0.1 x_{12, t-1}-0.4 x_{21, t-1}-0.2 x_{22, t-1} \\
& +0.3 x_{31, t-1}+0.4 x_{32, t-2}+\eta_{11, t} \\
x_{12, t}= & 0.4 x_{11, t-1}-0.2 x_{12, t-1}+0.1 x_{21, t-1}-0.4 x_{22, t-1} \\
& -0.2 x_{31, t-1}+0.3 x_{32, t-2}+\eta_{12, t} \\
x_{21, t}= & 0.3 x_{11, t-1}+0.4 x_{12, t-1}-0.2 x_{21, t-1}+0.1 x_{22, t-1} \\
& -0.4 x_{31, t-1}-0.2 x_{32, t-2}+\eta_{21, t} \\
x_{22, t}= & -0.2 x_{11, t-1}+0.3 x_{12, t-1}+0.4 x_{21, t-1}-0.2 x_{22, t-1} \\
& +0.1 x_{31, t-1}-0.4 x_{32, t-2}+\eta_{22, t} \\
x_{31, t}= & -0.4 x_{11, t-1}-0.2 x_{12, t-1}+0.3 x_{21, t-1}+0.4 x_{22, t-1} \\
& -0.2 x_{31, t-1}+0.1 x_{32, t-2}+\eta_{31, t} \\
x_{32, t}= & 0.1 x_{11, t-1}-0.4 x_{12, t-1}-0.2 x_{21, t-1}+0.3 x_{22, t-1} \\
& +0.4 x_{31, t-1}-0.2 x_{32, t-2}+\eta_{32, t}
\end{aligned}\right.
$$

We consider 200 realizations of 10000 sample time points of (32). For each realization we will estimate the regression model by the approach introduced in (ii) of Property 1. We will calculate the F-norm of power spectrum matrix of $X_{i}, i=1,2,3$, reported in Fig. 7(a) from which it is clear that $X_{1}, X_{2}, X_{3}$ have almost same F-norm of power spectrum matrix for all frequencies and are with a peak at $f=39.5 \mathrm{~Hz}$. Based on the estimated regression model we first compute spectral BNC and conditional spectral BGC values. Then, we calculate the average values across all realizations and report the results in Fig. 7(b). The first column in Fig. 7(b) shows direct spectral BNC values from $X_{1}$ to $X_{1}$, from $X_{2}$ to $X_{1}$, and from $X_{3}$ to $X_{1}$. It is notable that spectral BNC values from $X_{1}$ to $X_{1}$, from $X_{2}$ to $X_{1}$, and from $X_{3}$ to $X_{1}$ all have similar curves with the same peak frequency of $39.5 \mathrm{~Hz}$ as the power spectrum shown in Fig. 7(a). On the contrary, the second column of Fig. 7(b) is the conditional BGC values (from $X_{2}$ to $X_{1}$, and from $X_{3}$ to $X_{1}$ ) which have different peak frequencies, which all are not at $39.5 \mathrm{~Hz}$. Therefore, the spectral BNC results are much more interpretable and convincing than conditional BGC results.

Hence, by this example, we can claim that, generally speaking, spectral BGC may not necessarily reveal the strength of true causality.

Example 3: Since NC or GC is a special case of BNC or $\mathrm{BGC}$, in this example we consider the simulation example of two univariate time series described in the first paragraph of simulation section [43] in order to provide more evidence to demonstrate spectral $\mathrm{NC}$ or $\mathrm{BNC}$ is much better than spectral GC or BGC to reveal true causality. According to the description the two channels have event-related potentials (ERPs) produced by one cycle of $12 \mathrm{~Hz}$ sinusoidal waves which are combined with ongoing activities. The single-trial amplitudes of the sinusoidal wave for Channel 1 are chosen independently in the interval $[2,12]$. The single-trial amplitude for Channel 2 is the amplitude of Channel 1 plus standard Gaussian noise. The single-trial latency shifts for the ERP components are also considered and uniformly distributed between $0 \mathrm{~ms}$ and $10 \mathrm{~ms} .500$ trials (realizations), each with 120 data points at a sampling rate of $200 \mathrm{~Hz}$, are generated. Each trial is $600 \mathrm{~ms}$ long, $100 \mathrm{~ms}$ of which occurred prior to stimulus onset $(0 \mathrm{~ms})$. The ERP for Channel 1 starts about $50 \mathrm{~ms}$ after the stimulus onset, while the ERP from Channel 2 is delayed 


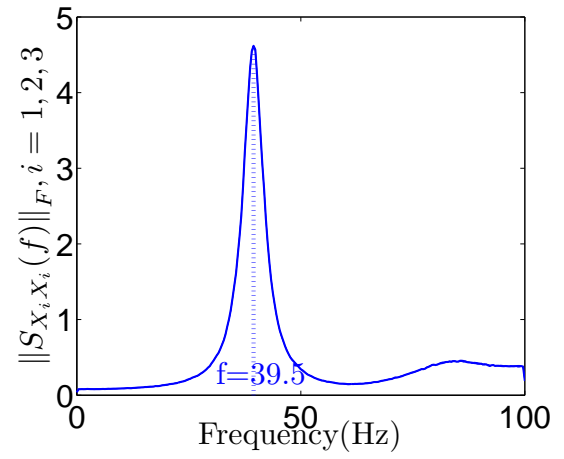

(a)

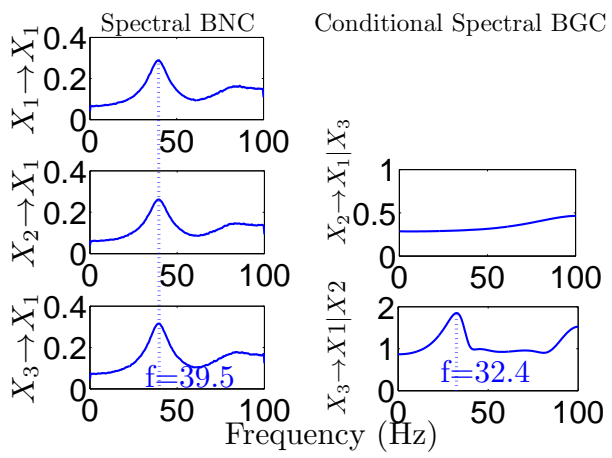

(b)

Fig. 7. (a) F-norm of power spectrum matrix of $X_{i}(i=1,2,3)$ with a same peak frequency at $f=39.5 \mathrm{~Hz}$. (b) Spectral BNC results and conditional spectral BGC results.

by about $20 \mathrm{~ms}$. The ongoing activity for both channels is Gaussian white noise processes with zero mean and 0.05 standard deviation. These two noise processes are uncorrelated with each other. Fig.8(a) shows the 500 simulated realizations (trials) for both channels. Since each ERP component involves one cycle of sinusoidal wave with ongoing activities of small amplitude, the former half cycle and the later half cycle in each ERP component have almost equal amplitude as seen in Fig.8(a). However, it is surprisingly noted that for each ERP component in Figs.2(a) and (b) [43], the amplitude of the former half cycle is always much smaller than that of the later half cycle, so the whole cycle is not a sinusoidal wave at all. This violates the requirement of sinusoidal waves in ERP components. Therefore, based on the description in the first paragraph of simulation section [43], we can only plot Fig.8(a) and cannot plot Figs.2(a) and (b) [43]. Next, we discuss causality issue only based on Fig.8(a). Since by construction, Channel 2 lags behind Channel 1 by 20ms, and the amplitudes of the two channels are assumed to be correlated on a trial-bytrial basis, there must exist a causal influence from Channel 1 to Channel 2 and no causal influence from Channel 2 to Channel 1.

To apply spectral GC and NC, we first obtain the average values (ave1 and ave2) of Channels 1 and 2 in each single trial, subtract ave1 (ave2) from Channel 1 (Channel 2), and get the residual data in Fig.8(b). This ensures the zero-mean requirement for both channels in each single trial. We then apply spectral GC and NC on the residual data. To study event-related causality between Channel 1 and Channel 2, we use moving window technology with the window size of 10 samples (50ms) and overlap of 2 samples (10ms). Due to the lags of $20 \mathrm{~ms}$ ( 4 sample points) between the two ERP components in each single trial, the optimal order for the regression model is chosen to be 6 over all windows in each single trial. Fig. 8(c) shows the time-frequency power spectra of both channels which are got by averaging over all trials. The figure clearly indicates the delayed (about $55 \mathrm{~ms}$ for Channel 1 and $75 \mathrm{~ms}$ for Channel 2 after stimulus onset by considering average $5 \mathrm{~ms}$ latency shift since the single-trial latency shifts are uniformly distributed between $0 \mathrm{~ms}$ and 10ms) enhanced lower frequency band $(<12 \mathrm{~Hz})$ activities. Figs. 8(d) and (e) describe the time-frequency causality flow (i.e., event-related causality flow) between Channel 1 and Channel 2 in terms of spectral GC and NC, respectively. From Figs. 8(d) and (e), we can clearly see that i) $\mathrm{NC}$ results demonstrate that there is stronger causal influence from Channel 1 to Channel 2 in lower frequency band $(<10 \mathrm{~Hz})$ and at about $55 \mathrm{~ms}$ after the stimulus onset which are consistent with power spectral results in Fig. 8(c), and there is no causal influence from Channel 2 to Channel 1 in any frequency and at any time instant. These results are true by above construction of both channels; ii) GC results demonstrate that there is stronger causal influence from Channel 1 to Channel 2 not only in lower frequency band $(<10 \mathrm{~Hz})$ but also in the frequency band $(20 \sim 25 \mathrm{~Hz})$ which is not consistent with the fact that no enhanced power can be found in the frequency band in Fig. 8(c). The time instant at which the enhanced causal influence takes place is before $50 \mathrm{~ms}$ where ERP components has not appeared yet, this is obviously not true. Moreover, there is stronger causal influence from Channel 2 to Channel 1 in lower frequency band $(<5 \mathrm{~Hz})$ before $50 \mathrm{~ms}$. This result is definitely not true by above construction of both channels. Therefore, by moving window technology, we clearly show that NC results are much correctly reveal true causality between Channel 1 and Channel 2 than GC results.

To show the detailed causal influence in frequency domain, we first plot power spectra of two channels in Fig. 9(a) or Fig. 9(b) where the power amplitude is shown in dB. From Fig. 9(a) one can clearly see that the power amplitudes for the frequency range $(>20 \mathrm{~Hz})$ is very small and for both channels there is the same peak at $10 \mathrm{~Hz}$ which surely comes from ERP components. From Fig. 9(b) one can clearly see that for both channels there are more three peaks at three different higher frequencies beside the peak at $10 \mathrm{~Hz}$. These peaks surely comes from ERP components. To calculate spectral GC and NC between Channel 1 and Channel 2, the optimal order of the regression model is chosen to be 5 ( $>4$, the delayed sample points due to the fact that Channel 2 lags behind Channel 1 by 20ms). NC and GC results are shown in Figs. 9(c) and (d) respectively. $\mathrm{NC}$ results in Fig. 9(c) demonstrate that there is the strongest causal influence from Channel 1 to Channel 2 at $10 \mathrm{~Hz}$ and causality curve from Channel 1 to Channel 2 is consistent with the power spectrum curves in Fig. 9(b). The causal influence from Channel 2 to Channel 1 is very small over all frequencies and can be ignored compared to 
larger causality from Channel 1 to Channel 2 . These results are surely true by construction of both channels. GC results in Fig. 9(d) demonstrate that there is strong causal influence (a peak can be seen at $11.7 \mathrm{~Hz}$ ) from Channel 1 to Channel 2 over all frequencies and causality curve from Channel 1 to Channel 2 is not consistent with the power spectrum curves in Fig. 9(b) at all, although the causal influence from Channel 2 to Channel 1 is very small over all frequencies and can be ignored compared to larger causality from Channel 1 to Channel 2 . Therefore, in frequency domain, we once again demonstrate that NC method is much better to reveal true causality than GC method.

Example 4: In this example we consider Example 5 [30]. The EEG recording is from the patient suffering from seizure in left temporal lobe (see the detail for the ERP experiment described in [30]). The EEG recording includes 8 right and 8 left temporal depth electrode channels. Their ERP images are plotted in Fig. 10 and clearly show cortical activity changes after stimulus onset at most of channels where we use the average referenced iEEG. To study event-related causality between different blocks, as in [30] we use moving window technology with the window size of 150 samples $(240 \mathrm{~ms})$ and overlap of 75 samples $(120 \mathrm{~ms})$ and select the regression model order of 8 for all windows in our data set.

In [30] we calculated causal influence between two univariate channels (L4 and R5) by using spectral NC, GC, PDC and RPC methods. The results demonstrated that spectral NC results are much more advantageous over GC, PDC and RPC by the fact that only spectral $\mathrm{NC}$ results have the similar enhanced frequency band $(<8 \mathrm{~Hz})$ as $\mathrm{R} 5$ and $\mathrm{L} 4$ shown in Fig. 16(b) in [30] from which one can clearly see the obvious delayed lower frequency band $(<8 \mathrm{~Hz})$ activities after stimulus onset (about $250 \mathrm{~ms}$ later). These results are consistent with existing findings that theta $(4 \sim 7 \mathrm{~Hz})$ band oscillations are prominent phenomena in cognitive processes of human and animal investigations [44]-[46]. This may play a vital role in memory consolidation and memory encoding [47]-[49].

Now let $\mathrm{R}=\{\mathrm{R} 4, \mathrm{R} 5\}$ and $\mathrm{L}=\{\mathrm{L} 4, \mathrm{~L} 5\}$ be two blocks of channels, we will take $\mathrm{R}$ and $\mathrm{L}$ as an illustrative example. According to spectral BNC and spectral BGC methods, we calculate blockwise causality between two blocks of $\mathrm{R}$ and $\mathrm{L}$. Fig. 11(a) and Fig. 11(b) describe the power spectra of L4, L5, R4, R5 which are got by averaging over all trials. The figures clearly demonstrate the delayed (about $250 \mathrm{~ms}$ after stimulus onset) enhanced lower frequency band $(<8 \mathrm{~Hz})$ activities. Fig. 11(c) describes the F-norm of power spectrum matrix of $\mathrm{R}$ or $\mathrm{L}$ which are got by averaging over all trials. The figure also clearly indicates the similar delayed enhanced activities. Fig. 12(a) and Fig. 12(b) describe the time-frequency causality flow (i.e., event-related causality flow, please refer to [17] for more details) between $\mathrm{R}$ and $\mathrm{L}$ in terms of spectral $\mathrm{BNC}$ and BGC, respectively. From these two figures (Fig. 12(a) and Fig. 12(b)), we can derive two important findings.

The first finding is that the causal influence from $\mathrm{R}$ to $\mathrm{L}$ revealed by both measures is significantly enhanced after stimulus onset (about $250 \mathrm{~ms}$ delay). Please note, in order to test the significance level for each measure, we employ the statistical testing framework introduced in [17]. However,

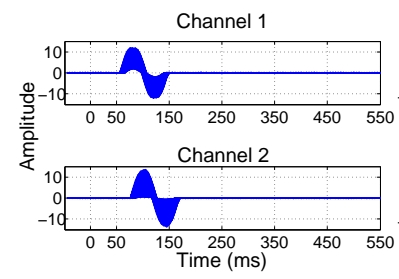

(a)

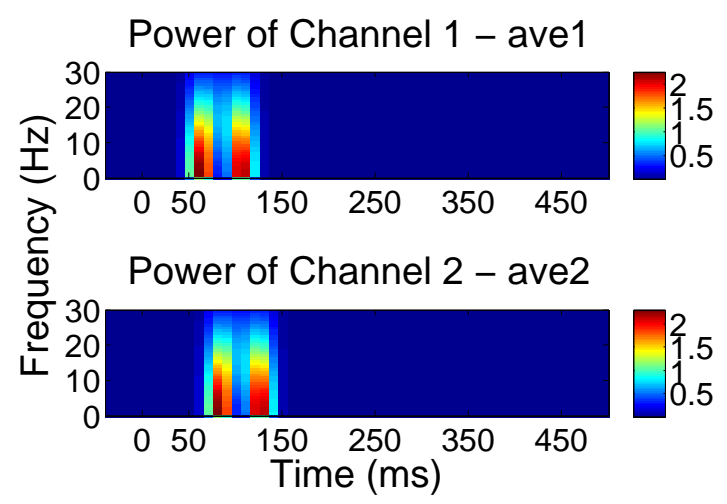

(c)

\section{GC: Channel 1 to Channel 2}

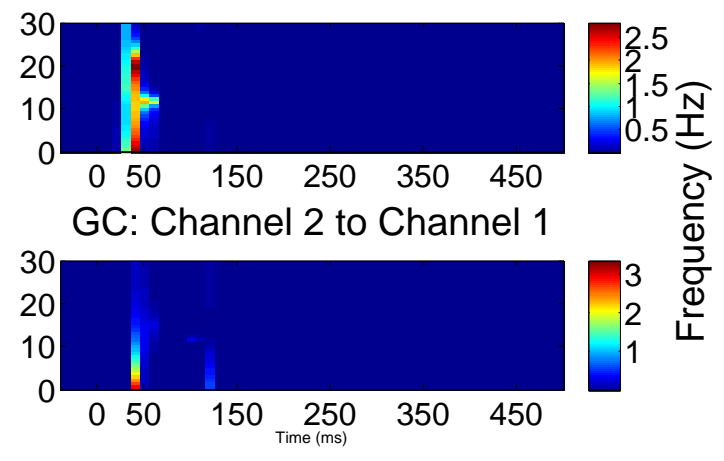

(d)

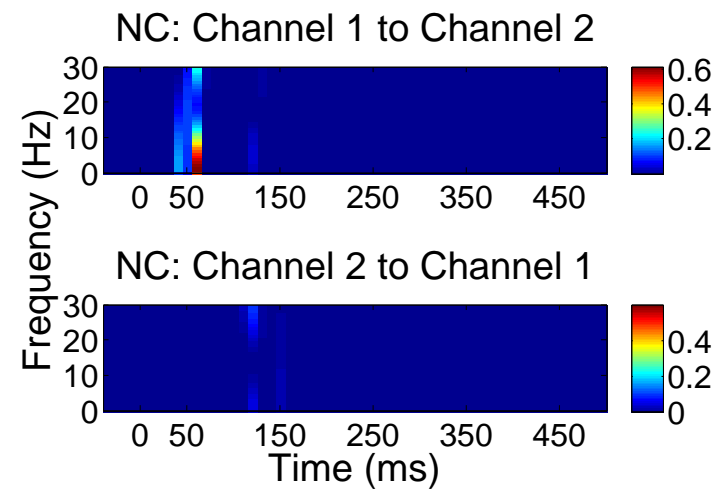

(e)

Fig. 8. (a) 500 realizations (trials) of simulated data for Channel 1 and Channel 2. Both latency variability and amplitude variability are considered. (b) Residuals after subtracting their own average values (of each single trial) from each single trial for both channels. (c) Time-frequency power spectrum for two channels (residuals). (d) Time-frequency new causality between Channel 1 and Channel 2 (residuals). (e) Time-frequency Granger causality between Channel 1 and Channel 2 (residuals). 


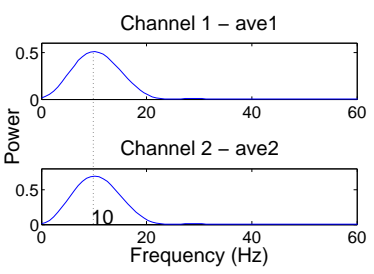

(a)

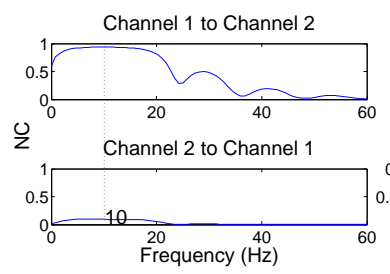

(c)

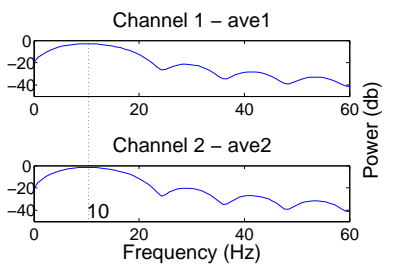

(b)

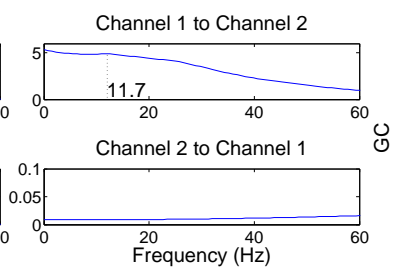

(d)
Fig. 9. (a) Power spectrum for two channels (the residual data in Fig. 8(b)). (b) Power spectrum in db for two channels. (c) Spectral NC between two channels. (d) Spectral GC between two channels.

only spectral BNC discloses the enhanced frequency band $(<$ $8 \mathrm{~Hz})$. This situation is consistent with the delayed enhanced activities of $\mathrm{R}$ and $\mathrm{L}$ demonstrated in Fig. 11(a) and Fig. 11(b). Therefore, the result should be convincing and true. The spectral BGC method demonstrates that the enhanced frequency band is less than $2 \mathrm{~Hz}$ and is not the enhanced band $(<8 \mathrm{~Hz})$ shown in Fig. 11(a) and Fig. 11(b), as a result, we believe that the spectral BGC result leads to misinterpretation.

The second finding is that both methods verify the much small causal flow from $\mathrm{L}$ to $\mathrm{R}$ compared to that from $\mathrm{R}$ to $\mathrm{L}$. These results demonstrate the asymmetric interactions between the two blocks, that is, $\mathrm{R}$ has a much stronger directional interaction on $\mathrm{L}$. We believe, the major reason causing this phenomenon is some of brain functions in left temporal lobe were lost because the patient had a left temporal lobe epilepsy so that the information flow in left temporal lobe is kept from transmitting to right temporal lobe.

To summarize, by analyzing the neurophysiological data from one patient, we once again demonstrated that the spectral BNC method can get better satisfactory and convincing results than the spectral BGC method.

\section{CONCLUSiON}

In neuroscience, currently multi-channels of EEG recording and functional imaging play a more and more important role in studying network mechanisms for cognition or disease diagnosis. For example, we may be interested in causal flow among different subsets (or groups, blocks) of the brain areas. Multivariate BGC is an useful tool to reveal causal influences between two blocks of time series. Especially the spectral BGC can be used to study blockwise causal influences in a variety of frequencies when one performs cognitive or perceptual tasks. For three or more blocks of variables, conditional BGC can be applied to study causal interactions in both time and frequency domains.

In this paper, we extended NC definition in [30] to blockwise case. We first considered time domain and proposed BNC

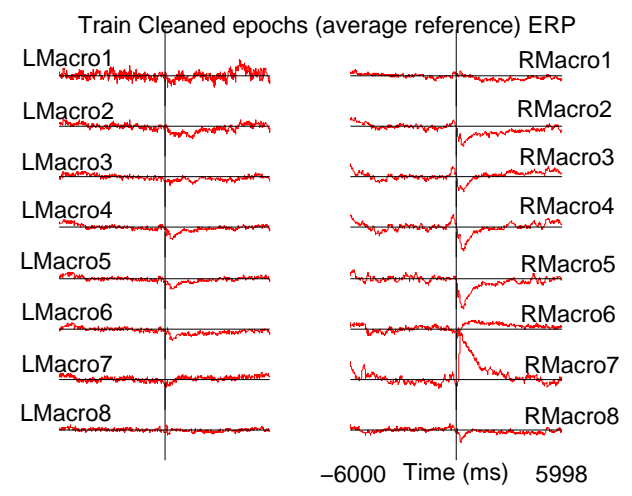

Fig. 10. ERPs for eight left Macrowire channels (LMacro1 LMacro8) and eight right Macrowire channels (RMacro1 RMacro8) where average reference is applied.

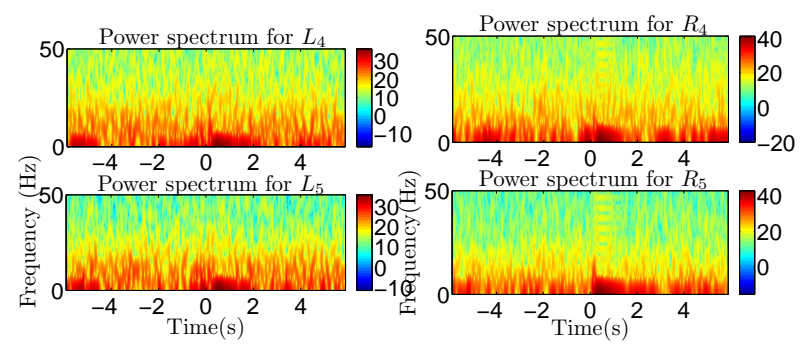

(a)

(b)

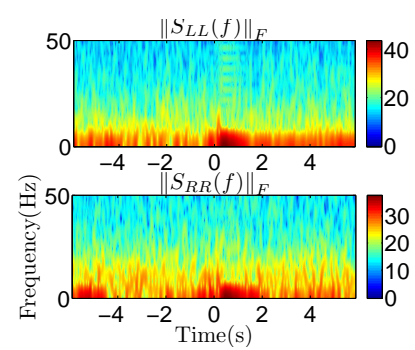

(c)

Fig. 11. (a), (b) The power spectra of L4, L5, R4 and R5. (c) The F-norm of power spectrum matrix of $\mathrm{R}$ (or $\mathrm{L}$ ).

definition from $X_{2}$ (one block of time series) to $X_{1}$ (the other block of time series) for the blockwise linear regression model. In BNC definition we considered all contributions to current $X_{1}$, viewed $X_{2}$ (its past values) as one of these contributions, and described the proportion that $X_{2}$ has among all these contributions. We pointed out that any contribution to current $X_{1}$ should be considered when introducing a good causality method, this is a general guideline for proposing any good causality method. Any causality method violating this guideline inevitably cannot reflect well the true causality or the trend of the true causality for two blocks of variables. BGC method violates this guideline and as a result may not disclose the true causal flow. So, one may make wrong interpretations only based on BGC value, such as, a bigger BGC value may not mean larger true causality; even in the case that BGC value is zero, true causality may still exist and can be obtained by BNC method. Therefore, one should be careful in drawing any conclusion only according to BGC 


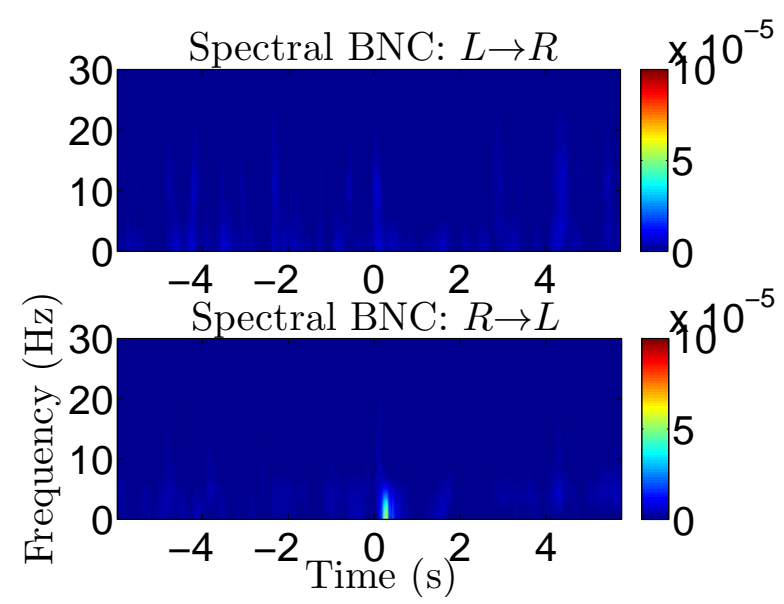

(a)

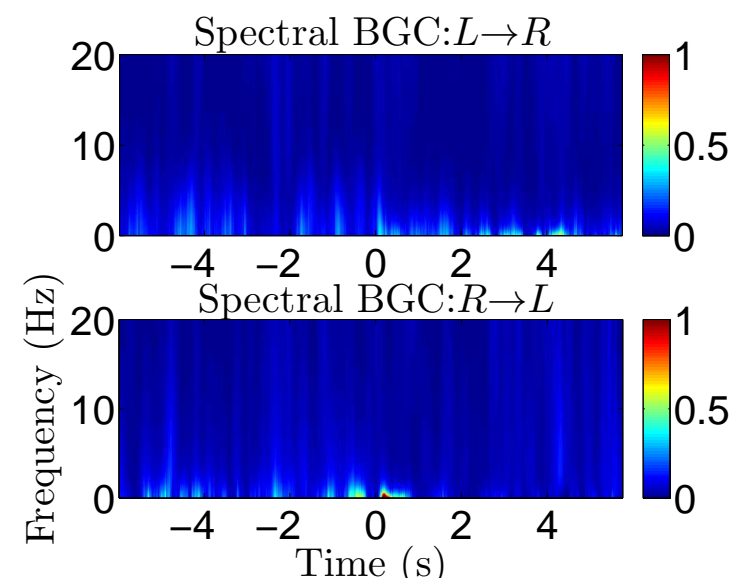

(b)

Fig. 12. (a), (b) Spectral BNC and BGC results between $\mathrm{L}$ and R, respectively.

value. We then discussed in frequency domain and pointed out that generally speaking, any causality definition according to the transfer function matrix or its inverse matrix of the linear regression model (frequency domain), such as, spectral BGC and conditional spectral BGC, may not disclose the true causality for two blocks of variables. To better disclose the true causality, we used a concept of proportion as in the time domain case and proposed the spectral BNC which describes how much proportion that one block of variables occupies among all contributions to another block of variables in the blockwise linear regression model (frequency domain). By several illustrative examples we demonstrated that our BNC definitions (in both of time and frequency domains) are more accurate and sensitive to reveal the true causality or the trend of true causality than the existing related definitions in the literature. Specifically, for a real EEG data from an epilepsy patient we applied the spectral BNC method and obtained satisfactory and convincing results. However, when we applied the spectral BGC method, the results were misleading. Thus, our BNC definitions as well as our previous NC definitions in [30] may have wide applications to reflect true causality among two blocks of variables or two univariate variables in economics, neuroscience and engineering.

\section{REFERENCES}

[1] D. N. Gujarati and D.C. Porter, "Causality in Economics: The Granger Causality Test," Basic Econometrics (Fifth international ed.). New York: McGraw-Hill. pp. 652 - 658, 2009.

[2] M.P. Chen, C.C. Lee, and Y.C. Hsu, "The impact of American depositary receipts on the Japanese index: do industry effect and size effect matter?" Econ. Model, vol. 28,pp. 526-539, 2011.

[3] K. Evan, C. Snigdhansu, and R.G. Auroop, "Exploring Granger causality between global average observed timeseries of carbondioxide and temperature," Theor. Appl. Climatol, vol. 104, pp. 325-335, 2011.

[4] J. Zhu, Y. Chen, A.S. Leonardson, K. Wang, J.R. Lamb, V. Emilsson, and E.E. Schadt, "Characterizing dynamic changes in the human blood transcriptional network," PLoS Comput. Biol. vol. 6, pp.e1000671, 2010.

[5] Q. Gao, X. Duan, and H. Chen, "Evaluation of effective connectivity of motor areas during motor imagery and execution using conditional Granger causality," NeuroImage, vol. 54, no. 2, pp. 1280-1288, 2011.

[6] T. Ge, J. Feng, F. Grabenhorst, and E. Rolls, "Componential Granger causality, and its application to identifying the source and mechanisms of the top-down biased activation that controls attention to affective vs sensory processing," Neuroimage, vol. 59, pp. 1846-1858, 2012.

[7] N. Wiener, The Theory of Prediction. In E.F. Beckenbach, editor, Modern Mathematics for Engineers, Chap. 8. McGraw-Hill, New York, 1956.

[8] C. W. J. Granger, "Investigating causal relations by econometric models and cross-spectral methods," Econometrica, vol. 37, no. 4, pp. 424-438, Aug.1969.

[9] J. Pearl, Causality: Models, Reasning and Inference, Cambridge University Press, 2009.

[10] P. Spirtes, C. Glymour, and R. Scheines. Causation, Prediction, and Search, Second Edition, The MIT Press, 2001.

[11] http://www.nobelprize.org/nobel_prizes/economic-sciences/laureates /2003/granger-lecture.html

[12] M. Ding, Y. Chen, and S. L. Bressler, "Granger causality: Basic theory and applications to neuroscience," in B. Schelter, M. Winterhalder, \& J. Timmer (Eds.), Handbook of Time Series Analysis. Weinheim: WileyVCH, pp. 437-460, 2006.

[13] J. Geweke, "Measures of conditional linear dependence and feedback between time series," J Am Stat Assoc, vol. 79, no. 388, pp. 907-915, Dec. 1984.

[14] L. A. Baccal and K. Sameshima, "Partial directed coherence: A new concept in neural structure determination," Biol Cybern, vol. 84, no. 6 , pp. 463-474, Jun. 2001.

[15] O. Yamashita, N. Sadato, T. Okada, and T. Ozaki, " Evaluating frequency-wise directed connectivity of BOLD signals applying relative power contribution with the linear multivariate time-series models," Neuroimage, vol. 25, no. 2, pp. 478-490, Apr. 2005.

[16] M. Kaminski, M. Ding, W. Truccolo-Filho, and S. L. Bressler, "Evaluating causal relations in neural systems: Granger causality, directed transfer function and statistical assessment of significance," Biol Cybern, vol. 85 , pp. $145-157,2001$.

[17] A. Korzeniewska, C. M. Crainiceanu, R. Kus, P. J. Franaszczuk, and N. E. Cronel, "Dynamics of event-related causality in brain electrical activity," Human Brain Mapping, vol. 29, no. 10, pp. 1170-1192, Oct. 2008.

[18] A. Brovelli, M. Ding, A. Ledberg, Y. Chen, R. Nakamura, and S. L. Bressler, "Beta oscillations in a large-scale sensorimotor cortical network: Directional influences revealed by Granger causality," in Proc. of the National Academy of Sciences of the United States of America, vol. 101, no. 26, pp. 9849-9854, 2004

[19] D. W. Gow, C. J. Keller, E. Eskandar, N. Meng, and S. S. Cash, "Parallel versus serial processing dependencies in the perisylvian speech network: A Granger analysis of intracranial EEG data," Brain and Language, vol. 110, no. 1, pp. 43-48, Jul. 2009.

[20] S. Guo, J. Wu, M. Ding, and J. Feng, "Uncovering interactions in the frequency domain," Plos computational Biology, vol. 4, no. 5, e1000087:1-5, 2008.

[21] H. Oya, P. W. F. Poon, J. F. Brugge, R. A. Reale, H. Kawasaki, and I. O. Volkov, "Functional connections between auditory cortical fields in humans revealed by Granger causality analysis of intra-cranial evoked potentials to sounds: Comparison of two methods," Biosystems, vol. 89, pp. 198-207, 2007. 
[22] J. R. Sato, D. Y. Takahashi, S. M. Arcuri, K. Sameshima, P. A. Morettin and L. A. Baccal,"Frequency domain connectivity identification: An application of partial directed coherence in fMRI," Human Brain Mapping, vol. 30, no. 2, pp. 452-461, Feb. 2009.

[23] B. Schelter, J. Timmer, and M. Eichler, "Assessing the strength of directed influences among neural signals using renormalized partial directed coherence," J. of Neuroscience Methods,vol. 179, no. 1, pp. 121-130, Apr. 2009.

[24] M. Kaminski and H. Liang, "Causal influence: advances in neurosignal analysis," critical reviews in biomedical engineering, vol. 33, no. 4, pp. 347-430, 2005.

[25] F. He, S. A. Billings, H. Wei, and P. G. Sarrigiannis, "A nonlinear causality measure in the frequency domain: Nonlinear partial directed coherence with applications to EEG," Journal of Neuroscience Methods, vol. 225,pp. 71-80, Mar. 2014.

[26] A. Papana, D. Kugiumtzs, and P. G. Larsso "Detection of direct causa effects and application to epileptic electroencephalogram analysis," International Journal of Bifurcation and Chaos, vol. 22, no. 9, pp. 1250222:1-7, 2012.

[27] W. H. Shim, K. Baek, J. K. Kim, Y. Chae, J. Suh, B. R Rosen, J. Jeong, and Y. Ro Kim, "Frequency Distribution of Causal Connectivity in Rat Sensorimotor Network: Resting-State fMRI Analyses," Journal of Neurophysiology, vol. 109, no. 1, pp. 238-248, Jan. 2013.

[28] A. Fasoula, Y. Attal, D. Schwartz, "Comparative performance evaluation of data-driven causality measures applied to brain networks," Journal of Neuroscience Methods, vol. 215, no. 2, pp. 170-189, 2013.

[29] G. Michalareas, J. Schoffelen, G. Paterson, J. Gross, "Investigating causality between interacting brain areas with multivariate autoregressive models of MEG sensor data," Human Brain Mapping, vol. 34, no. 4, pp. 890-913, Apr. 2013.

[30] S. Hu, G. Dai, G. A. Worrell, Q. Dai, and H. Liang, "Causality analysis of neural connectivity: critical examination of existing methods and advances of new methods," IEEE Trans. Neural Netw., vol. 22, no. 6, pp. 829-844, June. 2011.

[31] S. Hu, H. Wang, J. Zhang, W. Kong, Y. Cao, and R. Kozma, "Comparison Analysis: Granger Causality and New Causality, and their applications to Motor Imagery," IEEE Trans. Neural Netw. Learn. Syst., 16 pages, 2015, DOI: 10.1109/TNNLS.2015.2441137.

[32] A. B. Barrett and L. Barnett, "Granger causality is designed to measure effect, not mechanism," Frontiers in Neuroinformatics, vol. 7, Article 6, pp. 1-2, Apri. 2013.

[33] K. Imai, D Tingley, and T. Yamamoto,'Experimental designs for identifying causal mechanisms," J. R. Statist. Soc. A, vol. 176, Part 1, pp. 1-47, 2013.

[34] T. Ge, K. M. Kendrick, and J. Feng, "A Novel Extended Granger Causal Model Approach Demonstrates Brain Hemispheric Differences during Face Recognition Learning," Plos computational biology, vol. 5, no. 11, pp.e1000570:1-13, 2009.

[35] G. Sugihara1, R. May, H. Ye, C. Hsieh, E. Deyle, M. Fogarty, and S. Munch, "Detecting Causality in Complex Ecosystems," Science, vol. 338, no. 6106, pp. 496-500, Oct. 2012.

[36] J. Geweke, "Measurement of linear-dependence and feedback between multiple time series," Journal of the American Statistical Association, vol. 77, no. 378, pp. 304-313, Jun. 1982.

[37] Z. Zhou, Y. Chen, M. Ding, P. Wright, Z. Lu, and Y. Liu, "Analyzing brain networks with PCA and conditional Granger causality," Hum. Brain Mapp vol. 30, pp. 2197-2206, 2009.

[38] Y. Chen, S. L. Bressler, and M. Ding, "Frequency decomposition of conditional Granger causality and application to multivariate neural field potential data", J. Neurosci. Methods, vol. 150, pp. 228-237, 2006.

[39] X. Wen, G. Rangarajan, and M. Ding, "Multivariate Granger causality: an estimation framework based on factorization of the spectral density matrix," Phil. Trans. R. Soc. A, vol. 371, no. 1997, pp. 20110610, 2013.

[40] X. Wang, Y. Chen, S. L. Bressler, and M. Ding, "Granger causality between multiple interdependent neurobiological time series: blockwise versus pairwise methods," International Journal of Neural Systems, vol. 17, no. 2, pp. 71-78, Apr. 2007.

[41] A. B. Barrett, L. Barnett, and A. K. Seth, "Multivariate Granger Causality and Generalized Variance," Physical Rev E, vol. 81, pp. 041907, 2010.

[42] Y. Chen, S. L. Bressler, and M. Ding, "Frequency decomposition of conditional Granger causality and application to multivariate neural field potential data," Journal of Neuroscience Methods, vol. 150, no. 2, pp. 228-237, Aug. 2006.

[43] X. Wang, Yong. Chen, and M. Ding, "Estimating Granger causality after stimulus onset: A cautionary note," Neuroimage, vol. 41, pp. 767-778, 2008.
[44] K. L. Anderson, R. Rajagovindan, G. A. Ghacibeh, K. J. Meador, and M. Ding, "Theta oscillations mediate interaction between prefrontal cortex and medial temporal lobe in human memory," Cerebral Cortex, vol. 20, no. 7, pp. 1604-1612, Jul. 2010.

[45] X. Xu, L. An, X. Mi, and T. Zhang, "Impairment of Cognitive Function and Synaptic Plasticity Associated with Alteration of Information Flow in Theta and Gamma Oscillations in Melamine-Treated Rats," Plos One, Oct. 2013, doi:10.1371/journal.pone.0077796.

[46] D. Hwanga and A. Golby, "The brain basis for episodic memory: insights from functional MRI, intracranial EEG, and patients with epilepsy," Epilepsy \& Behavior, vol. 8, no. 1, pp. 115-126, Feb. 2006.

[47] S. Raghavachari, J. Lisman, M. Tully, J. Madsen, E. Bromfield, and M. Kahana, "Theta oscillations in human cortex during a working-memory task: Evidence for local generators," Journal of Neurophysiology, vol. 95, no. 3, pp. 1630-1638, Mar. 2006.

[48] F. Roux and P. J. Uhlhaas, "Working memory and neural oscillations: alpha - gamma versus theta - gamma codes for distinct WM information?" Trends in Cognitive Sciences, vol. 18, no. 1, pp. 16-25, Nov. 2013.

[49] E.t Mas-Herrero and J. Marco-Pallares, "Frontal theta oscillatory activity is a common mechanism for the computation of unexpected outcomes and learning rate," Journal of Cognitive Neuroscience, vol. 26, no. 3, pp. 447-458, 2014. 